\title{
Towards a sustainable system: application of temporal analysis on flood risk management
}

\author{
Z. Alsaqqaf \& H. Zhang \\ Griffith School of Engineering, Griffith University, Australia
}

\begin{abstract}
The escalating frequencies and changing patterns of climate change impacts, such as precipitation rates and sea levels, question the reliability of the existing engineering infrastructure, in terms of design and planning criteria for which designers and decision makers need to or account for. The objective of this paper is to assess the performance of an existing engineering infrastructure by measuring three variables: Vulnerability $(\beta)$, Reliability $(\alpha)$, Resiliency $(\gamma)$. These variables will be implemented temporally to a floodplain catchment, where performance and engineering sustainability can be depicted. The depiction will define the system's behaviour upon a natural event such as precipitation or sea-level rise. Nevertheless, Flood Risk Index (FRI), which depends on ( $\beta, \alpha$ and $\gamma$ ), will be applied as an overall index to demonstrate the trend context as well as give implications of the sensitivity significance of $\beta, \alpha$ and $\gamma$. The main outcome of this paper is to depict the relative sustainability or as known as the performance assessment indicators temporally; and to examine the correlation between the indicators on a real-flow data. These procedures shall ultimately provide implications on the implementation of the indicators to achieve a relatively sustainable system.
\end{abstract}

Keywords: reliability, vulnerability, resiliency, flood risk index, sustainability, performance assessment.

\section{Introduction}

In the last decade, more frequent storms and sea level rises have been observed and monitored in Australia due to climate change especially southeast Queensland; which results in the increase of floods in many areas that already prone to flood. Consequently, new floodplains will emerge to cope with such an 
impact, placing assets like humans and properties at risk. The escalating impact of climate change in Australia makes it necessary to reassess existing flood protection systems and to assess the need for new ones in areas that were not previously at risk from floods.

In flood prone areas, having an adequate flood protection system in place is essential to lives and livelihood of residents and businesses in the area. In addition, catchments in coastal areas are prone of flooding due to cyclones and severe storms. In face of increased flood potential due to climate change, it is essential that the current system of structural flood inhibitors be examined to make certain that they offer the maximum protection for the predicted future flood scenarios. One of the examination tools that can be used is performance assessment.

The purpose of this paper is to assess the performance of a floodplain catchment. In a way, to depict the behaviour of the system under a flood event temporally. The paper will examine the feasibility of applying the performance assessment, or as known as the engineering relative sustainability indices, which are: Vulnerability $(\beta)$, Reliability $(\alpha)$, Resiliency $(\gamma)$, as well as an overall index called Flood Risk Index (FRI).

Sustainability plays an important role in the advancement of modern societies. it has become an inevitable factor in enhancing the implementation of an efficient strategic development and planning. Consequently, governments and city councils start to merge sustainability in their existing and future visions. In addition, many scholars have illustrated a constructive argument on a feasible definition of sustainability. Yet, they all hover under the umbrella of a continuous validity of a healthy development that perpetuates a long life status without confronting the needs of future generations as illustrated in many studies [1-3].

Engineering sustainability, as the name implies, is a characteristic of an engineering system that provides a self maintained robust system, which assures a quality standard for the demanded service and overcomes the deterioration factors that influences the engineering system over time [4].

Ultimately, it should lead to a different type of approach that will assist in solving current and future challenges on the engineering infrastructure. Hence, it is necessary that the performance of an engineering system constantly tested and evaluated to cope with such challenges. One method can quantitatively measure the engineering sustainability called relative sustainability or as known as the performance assessment indicators of an engineering system [5]. Similarly, according to Loucks [1], there are three commonly used indicators to measure engineering relative sustainability: reliability, vulnerability and resiliency. In other words, they provide the reflective behaviour of a system under defined impact or influence.

Basically, so far, these indicators were measured subjectively. However, this paper is in the first phase of rallying towards measuring these indicators objectively and accurately than the predecessor methodologies in the sustainability or performance assessment filed. Moreover, this paper is intending to implement the engineering relative sustainability, or as known as performance 
assessment indicators, on a real-flow data temporally to test the behaviour of the indicators.

\section{Performance assessment and flooding}

According to one of the pioneer studies of engineering performance assessment (Hashimoto et al. [5]), there are three main indices that actually characterise the performance assessment of a system: reliability, vulnerability and resiliency.

The performance measurement of an engineering system has been described by Moy et al. [6] as the ability of a system to deliver the targeted demand. As an illustration, they have applied performance assessment on a water supply reservoir by measuring three performance indices, reliability, vulnerability and resiliency. Where, the ability to supply the needed target release is referred to as reliability. And, the maximum shortfall form the targeted release, referred to as vulnerability. Also, the number of consecutive periods of deficit is referred to as system resilience [6]. These three measurement parameters constitute the most common performance measures of a reservoir system.

Following the work of Hashimoto [5], there have been several applications in the criteria of performance assessment. Datta and Houck [7] have applied performance assessment on different problem areas in the water resources field. They have compared multiple operation strategies reflecting the relative importance of divergence from targets for reservoir release versus reservoir storage divergence. Then, Weeraratne et al. [8] applied the reliability, resiliency and vulnerability to estimate reservoir release polices corresponded to different target flow levels at failure or critical points in the system.

Nevertheless, Moy et al. [6] examined the trade-offs between reliability, resiliency and vulnerability in the context of reservoir operation for water supply using a multi-objective mathematical model. After that, Burn and Simonovic [9] have introduced an improved real-time operation model for reservoirs, that utilises the trade-off between flow predict reliability and the performance of the reservoir operation.

The performance goal of any reservoir system is to reduce the shortfall, resulting in decreased vulnerability. The greater farther the maximum shortfall; the greater the vulnerability. Likewise, the shorter the maximum length of deficits, the greater the resilience. Using mixed-integer linear programming, a trade-off was found between reliability, vulnerability, and resilience. As the reliability increases or the maximum length of consecutive shortfalls decreases, the vulnerability of the system to greater deficits in supply increases. These relationships are inversely proportional to some degree, depending on the system [6].

On a similar point of view, Simonovic et al. [10] have simply defined the three performance indicators: as "reliability is the likelihood of system failure, vulnerability is used to describe the severity of the failure and resiliency measures how quickly the system recovers from the failure state".

Scientists and researches up to the year 2000 have applied performance assessment to examine water supply reservoirs as part of the engineering infrastructure. However, Simonovic [11] has introduced the first application of 
performance assessment on flood protection systems. Then, followed by a collaborative work of Simonovic and Li [12] to establish a methodology in implementing performance assessment on flood protection systems under the effect of climate change and supported by system dynamics. After that, Elbaroudy and Simonovic [13] have enhanced a methodology to evaluate the performance of water resource system using fuzzy sets, as well as the performance indicators. Nevertheless, Ahmad and Simonovic [14] have applied the Geographic Information System (GIS) and fuzzy analysis to simulate the water resource system spatially and temporally. Finally, Simonovic [15] illustrated a new method for spatial and temporal analysis of risk in water resources management.

Apparently, all the previous benchmark researches mentioned above have set a profound and respectable foundation in evaluating and assessing the performance of a system. Yet, it exposed a gap in the compatibility between the phases of evaluation throughout their phases of methodology. In addition, there was a complication in defining the avenues of a system's failure status, and classifying the spectrum of the acceptable range in a system.

In summary, designing a failure free system is next to impossible. Even the best system designs are susceptible to fail. In the case of flood protection systems, it is difficult to predict the impacts that natural forces will place on the system. Even the best-designed system can be inundated by extremes from nature, causing ultimate system failure. Therefore, as a decision maker the performance assessment indicators should reveal the reliability, resiliency, vulnerability and FRI must be applied to examine the robustness of the existing systems, as well as it should be considered as an essential planning and designing factor not just for floodplain catchments, but for any engineering infrastructure system.

The terms reliability, resiliency, and vulnerability were typically applied to water supply systems including water reservoirs. Yet, the problem addressed by this paper is not one of supply, but one of deterrence. The system will be modelled and tested with the goal of impeding water from reaching communities and populations within the catchment. Developing a system that can accurately describe the ability of the flood deterrence system to prevent damage and injury to persons living in the flood plain, as well as human assets is the purpose of this paper. The same terms used to describe the reliability of a water supply system can be applied to a water deterrence (protection) system as well. All the performance indicators will be elaborated in the following sections.

\subsection{Reliability}

The reliability of a system can be defined as the satisfactory state. Also, it can be expressed as the probability that a system is operating in a satisfactory state at any given time. Inversely, the reliability can refer to the probability that no failure occurs within a given amount of time [5]. The reliability which is considered the opposite of risk is defined in eqn (1) as:

$$
\alpha=\frac{1}{\mathrm{~N}_{\mathrm{s}}} \sum_{i=1}^{\mathrm{N}_{\mathrm{s}}} z_{i}
$$


At the time $\mathrm{t}_{i}$,

$$
\begin{aligned}
& z_{i}=1, \text { if } x_{i}<x_{r e f} \\
& z_{i}=0, \text { if } x_{i} \geq x_{r e f}
\end{aligned}
$$

where $\alpha$ is the reliability. $\mathrm{z}_{i}$ is the state of the flood control system at the time $\mathrm{t}_{i}$, $x_{i}$ is the water level at time $\mathrm{t}_{i}, x_{\text {ref }}$ is the reference water level at time $\mathrm{t}_{i}$, and $\mathrm{N}_{\mathrm{S}}$ is the maximum number of time intervals.

For the present research, the failure state is considered to be at the time when the water level exceeds both catchment and river channel capacity on the entire grid of study domain. In other words, failure occurs when water depth exceeds the capacity depth of the catchment or river channel. Not to mention, $\mathrm{z}_{i}$ is a count function of $\mathrm{x}_{i}$ and $\mathrm{x}_{\text {ref }}$ [12].

\subsection{Vulnerability}

Vulnerability basically means measuring the extent of the failure. It is simply defined as the difference between the reference and calculated values of a certain variable including water depth [12]. This can be used to measure the overall impact that flood have on the overall functional ability of a flood protection system as shown in eqn (2).

For yearly basis at time $t_{i}$ :

$$
\beta_{y}= \begin{cases}0 & , x_{i} \leq x_{\text {ref }} \\ \operatorname{Max}\left[x_{i}-x_{\text {ref }}\right], & x_{i}>x_{\text {ref }}\end{cases}
$$

where $\beta_{y}$ is the vulnerability. In addition the statistical vulnerability is defined in eqn (3),

$$
\beta_{m}=\frac{\sum_{f=1}^{N_{f}} \beta_{y}}{N_{f}} \quad \text { and } \quad \beta_{n}=\frac{\sum_{f=1}^{N_{f}} \beta_{y}}{\mathrm{X}_{r e f} \cdot N_{f}}
$$

where $\beta_{m}$ is the mean vulnerability, $f$ is the counter of failure states, $\mathrm{N}_{f}$ is the total number of failure states during the operating period, and $\beta_{n}$ is the normalized mean vulnerability. As for this paper, the failure state will be all water level values $\left(\mathrm{x}_{i}\right)$ more than the catchment level $\left(\mathrm{x}_{r e f}\right)$.

\subsection{Resiliency}

Resiliency describes how quickly the system is likely to recover once a failure occurs [5]. In the event of a failure, it is important that a system is able to recover quickly and return to a state of stability. This can be expressed in eqn (4).

$$
\gamma=\frac{1}{\left(\frac{M}{N_{s}}\right) N_{f}}
$$

where $\gamma \dot{\mathbf{s}}$ the resiliency, $\mathrm{M}$ is the maximum number of consecutive time intervals of failure state in a year, $\mathrm{N}_{s}$ is the number of days in a year and, $\mathrm{N}_{f}$ is the number of failure state time intervals in a year. 
The application of the resilience equation will indicate the historical behaviour of the system. However, by plugging in climate change simulated and projected data, it should signify the expected behaviour in the future.

\subsection{Flood risk index (FRI)}

Zongxue et al. [16] have stated that FRI is an overall behaviour indicator that uses a weighted function of reliability, resiliency, and vulnerability to assess the flood risk potential for an area. It combines all three performance indicators in one equation as illustrated in eqn (5). This method takes into account, not only if a flood is likely in an area, but also how severe it may be and how long it may take to recover [16].

$$
P=\omega_{1} \cdot(1-\alpha)+\omega_{2} \cdot(1-\beta)+\omega_{3} \cdot \gamma
$$

in which

$$
\sum_{i=1}^{3} \omega_{i}=1.0
$$

where $\mathrm{P}$ is the flood risk index, $\alpha$ is the reliability, $\beta_{y}$ is the vulnerability, $\gamma$ is the resiliency and $\omega_{1}, \omega_{2}, \omega_{3}$ are predetermined weights. For the time being, it will be assumed that every predetermined weight is the same as in eqn (6).

\section{Case study}

The intention of this case study is to test the indicators on a real flow data to depict the behaviour of the performance assessment (relative sustainability) indices. Fig. 1 shows the trial daily flow data for a site in southeast Queensland, Australia. The daily flow data is dated from 1928 to 1960.

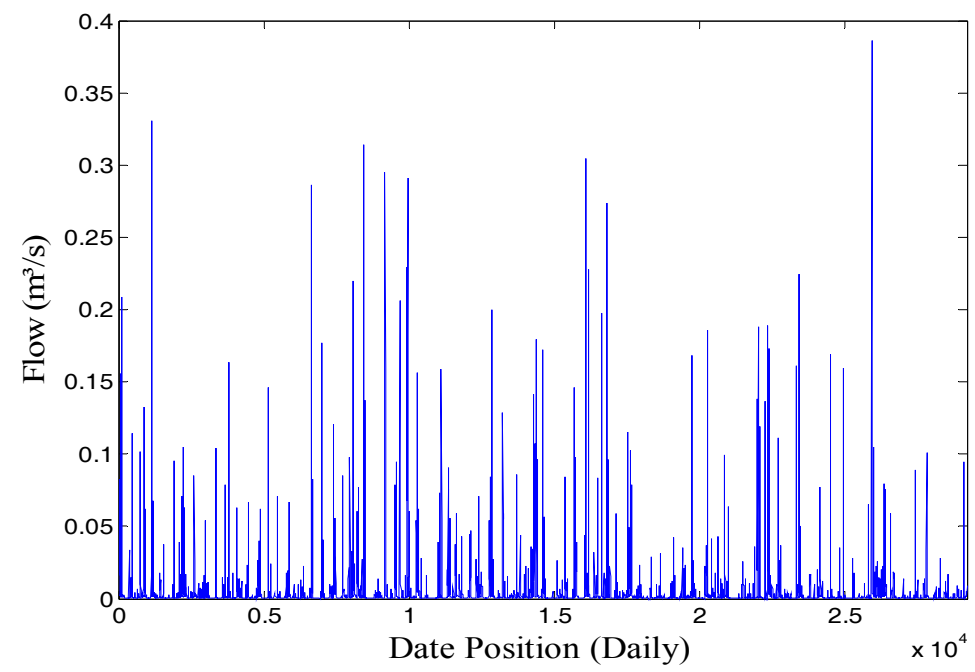

Figure 1: Daily flow data $\left(\mathrm{m}^{3} / \mathrm{s}\right)$. 
The calculations of the indicators were based on the flow data above. Fig. 2 shows the deduced values of the performance assessment indicators after the substitution for eqns (1), (2), (3), (4), (5), (6). It is notable that all the indicators have the results between 0 and 1 , which it can be also represented in a percentage form.

The reliability indicator as shown in fig. 2 demonstrates the satisfactory or no failure state in a given event. Explicitly, it behaves the opposite of the vulnerability indicator.
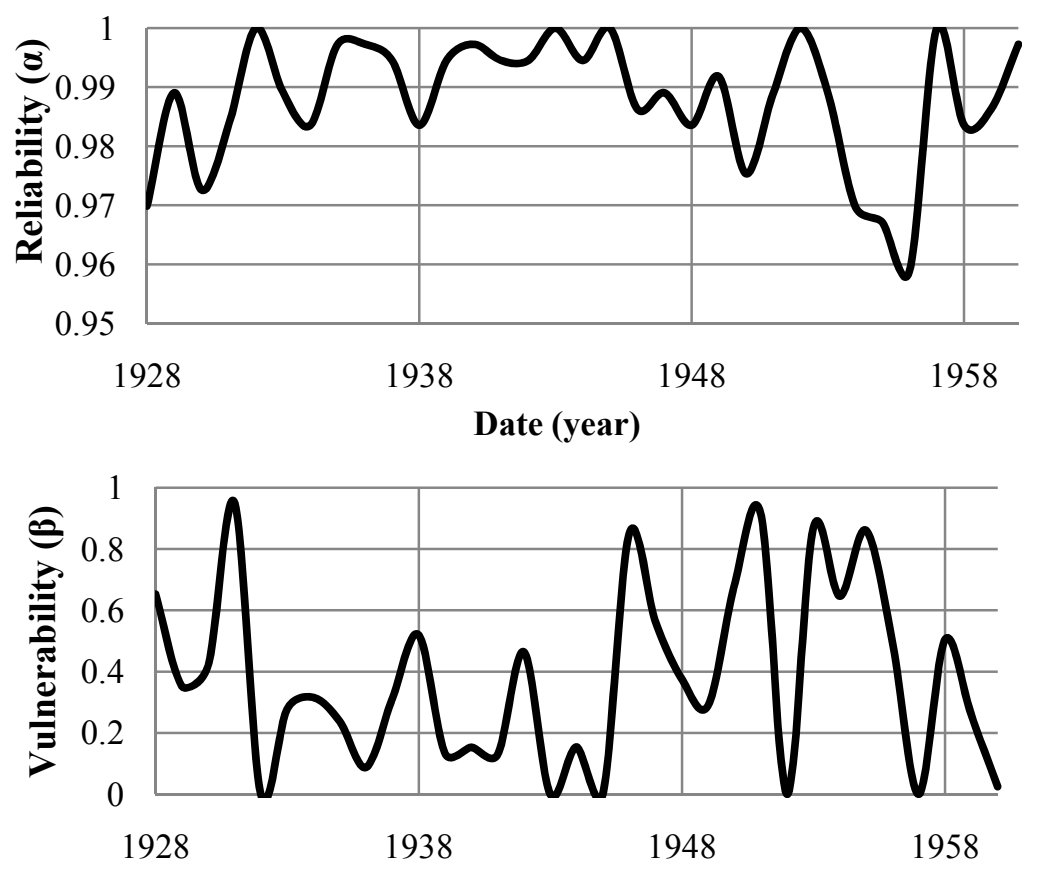

Date (year)

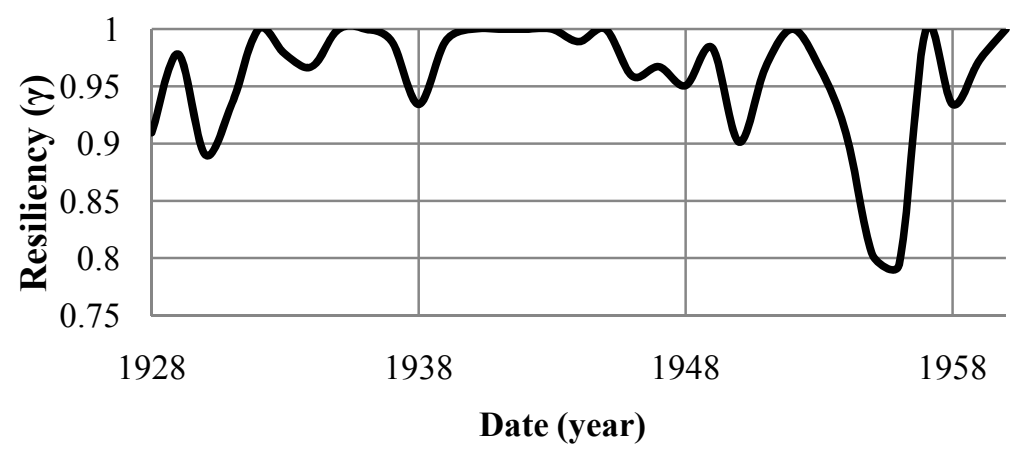

Figure 2: Performance assessment indicators. 
The representation of the performance assessment showed a consistent relationship with the flow data tested. For example, when looking at the dip of year 1955 on the reliability and resiliency trend, one can note that the vulnerability showed a spike in the same event. In the same time, the resiliency trend showed a significant sensitivity, due to a longer consecutive failing state.

Apparently, the indicators have behaved as expected. They have shown a proportional correlation between the water level and the vulnerability index as well as the FRI index. Also, they have shown an inverse correlation between the water level and the reliability and resiliency indices.

As a matter of fact, FRI illustrates a combined indicator of all of the three performance assessment indices as presented in fig. 3. The main objective of this indicator is firstly, to identify the sensitivity of each indicator. Secondly, to calibrate and verify the results according to the site studied. Thirdly, to set the acceptable risk ranges, which are the highest and lowest ranges that the decisionmaker deems to be acceptable.

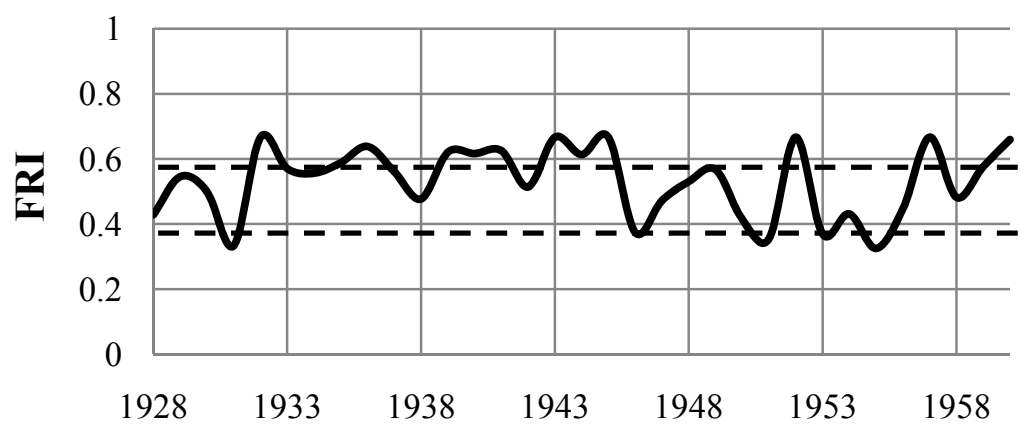

Acceptable Ranges

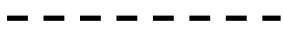

\section{Date (year)}

Figure 3: $\quad$ Flood risk index.

The acceptable risk ranges shown in fig. 3, depends on the definition of failure, perception of risk and the applied constrains such as budget available. Consequently, by setting these ranges, the indicator is capable of providing implications for flood risk management, in such an optimum plan or design can be achieved.

\section{Conclusion and future work}

This paper is the first part of a project series in implementing the performance assessment indicators to evaluate the sustainability of an engineering system as well as indentifying the risk significance in the case of flooding. However, the main purpose of this paper is to illustrate the risk incurred from an increase of the water level above the catchment level. And, to depict temporally the indicator's behaviour. 
The performance assessment (relative sustainability) indicators have been applied and tested on a real flow data. The results have shown a proportional correlation between the water level and the vulnerability, as well as the FRI index. Also, it showed an inverse correlation between the water level and the reliability and resiliency indicators.

Speaking of future work, it is intended to apply the performance assessment indicators temporally and spatially. It will involve a 2-dimontional hydraulic simulation to enhance the accuracy of the indicators, and to overcome limitations that involve subjectivity and ambiguity in identifying risk in an engineering system. Nevertheless, the spatial hydraulic simulation can accurately depict the extent of the risk or flood spatially, which in turn will identify the significance, and the sensitivity of the influencing elements that contributes to the risk paradigm.

\section{References}

[1] Loucks, D. P., Quantifying trends in system sustainability. Hydrological Sciences Journal, 42(4), pp. 513-530, 1997.

[2] Simonovic, S. P., Risk in sustainable water resources management. In: Sustainability of Water Resources Under Increasing Uncertainty: the Rabat Symposium. IAHS Publication, 240, pp. 3-17, 1997.

[3] Sahely, H. R., Kennedy, C. A., \& Adams, B. J., Developing sustainability criteria for urban infrastructure systems. Canadian Journal of Civil Engineering, 32, pp. 72-85, 2005.

[4] American Society of Civil Engineers (ASCE) and United Nations Scientific, Educational and Cultural Organization (UNESCO), Sustainability Criteria for Water Resource Systems, ASCE, Reston, Va., 1998.

[5] Hashimoto, T., Stedinger, J. R., \& Loucks, D. P., Reliability, resiliency, and vulnerability criteria for water resource system performance evaluation. Water Resour. Res., 18(1), pp. 14-20, 1982.

[6] Moy, W.-S., Cohon, J. L., \& ReVelle, C. S., A programming model for analysis of the reliability, resilience, and vulnerability of a water supply reservoir. Water Resour. Res., 22(4), pp. 489-498, 1986.

[7] Datta, B., and Houck, M. H., A stochastic optimization model for real-time operation of reservoirs using uncertain forecasts. Water Resource Research, 20, pp. 1039-1046, 1984.

[8] Weeraratne, J. R., Logan, L. And Unny, T. E., Performance evaluation of alternate policies on reservoir system operation. Canadian Journal of Civil Engineering, 13, pp. 203-212, 1986.

[9] Burn, D. H. And Simonovic, S, P., An improved methodology for short-term operation of a single multi-purpose reservoir. Water Resources Research, 25, pp. 1-8, 1989.

[10] Simonovic, S. P., Venema, H. D., \& Burn, D. H. 1992. Risk-based parameter selection for short-term reservoir operation. Journal of Hydrology, 131(1-4), pp. 269-291, 1992. 
[11] Simonovic, S. P., Assessment of the impact of climate variability and change on the reliability, resiliency and vulnerability of complex flood protection systems. Rep. to the Natural Resources Canada, London, Ontario, 2001.

[12] Simonovic, S. P., \& Li, L., Methodology for assessment of climate change impacts on large-scale flood protection system. Journal of Water Resources Planning and Management, 129(5), pp. 361-371, 2003.

[13] El-Baroudy, I., \& Simonovic, S. P., Application of the fuzzy performance measures to the city of London water supply system. Canadian Journal of Civil Engineering, 33, pp. 255-265, 2006.

[14] Ahmad, S. S., \& Simonovic, S. P., A methodology for spatial fuzzy reliability analysis. Applied GIS, 3(1), pp. 1-42, 2007.

[15] Simonovic, S., A new method for spatial and temporal analysis of risk in water resources management. Journal of Hydroinformatics, 11(3-4), pp. 320-329, 2009.

[16] Zongxue, X., Jinno, K., \& Kawamura, A. et al., Performance risk analysis for Fukuoka water supply system. Water Resources Management. 12, pp. 13-30, 1998. 